\title{
TABLE DES MATIÈRES DU TOME I
}

\section{LE SÉRIEUX DE L'INTENTION}

\author{
CHAPITRE PREMIER
}

\section{L'incertaine certitude des évidences morales}

I. L'Autre et l'Ego :

I. Confirmation de l'Affirmation (Préférabilité de l'Etre, du Plus-Etre)................. 4

2. Infirmation de l'Affrmation (Préférabilité de l'Altruisme) $\ldots \ldots \ldots \ldots \ldots \ldots \ldots \ldots \ldots \quad 7$

II. Paradoxologie de l'organe-obstacle :

3. Un Malgré qui est Parce-que............ Is

4. L'Amour et le sujet aimant.............. I9

5. Le Mérite. Pardon, Fidélité, Courage, Sincérité.. 25

III. L'Instant :

6. L'instant intentionnel clignote à travers le corps, la conscience et la continuation............. 30

7. Le Presque-rien et l'impossible-nécessaire : de l'Occasion .................... 38

IV. L'ambiguité et l'évidence :

8. Ampbibolie et réciprocité de l'égoïsme et de l'altruisme $\ldots \ldots \ldots \ldots \ldots \ldots \ldots \ldots .42$

9. Le mélange, le va-et-vient, l'équivoque infinie.... 47

10. Evidence inévidente de la liberté........... 52 
CHAPITRE II

\section{Du plaisir au logos}

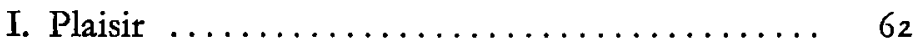

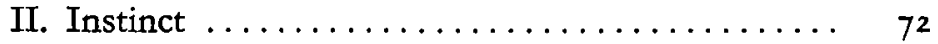

III. Eudémonie ..................... 77

IV. Ordo voluptatis . . . . . . . . . . . . $\quad 88$

V. Le scandale de la douleur................ IOI

CHAPITRE III

La douleur du devoir-faire

I. Devoir et Nature................... 106

II. Devoir et gratitude ................. I I 2

III. La surnaturalité morale $\ldots \ldots \ldots \ldots \ldots \ldots$ II

IV. Ce qui est fait reste à faire.............. I 25

V. De l'obligation, et de la grâce $\ldots \ldots \ldots \ldots \ldots \ldots{ }_{3} 6$

\section{CHAPITRE IV}

De la Conscience ou la douleur d'avoir-fait

I. Conscience de soi $\ldots \ldots \ldots \ldots \ldots \ldots \ldots$ I 43

II. Demi-conscience $\ldots \ldots \ldots \ldots \ldots \ldots \ldots \ldots \ldots$ I 47

III. Conscience morale $\ldots \ldots \ldots \ldots \ldots \ldots \ldots$ I $\ldots 3$

IV. Conscience douloureuse $\ldots \ldots \ldots \ldots \ldots \ldots \ldots$ I64

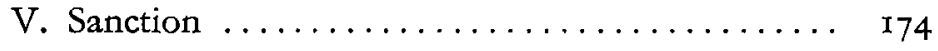

\section{CHAPITRE V \\ L'intention-de-faire}

I. Le futur intentionnel. .................. $\quad$ r80

II. L'aporie infernale ................. I94 
CHAPITRE VI

\section{Qu'il faut faire le Bien}

I. Quelque chose qu'il faut faire........... 217

II. C'est à moi de le faire................. 228

III. Tout moi; et du sérieux (Tout-ou-rien) ....... $\quad 236$

IV. Quelque chose que tout le monde peut faire..... 247

V. Qu'il faut le faire séance tenante (Tout-de-suite-

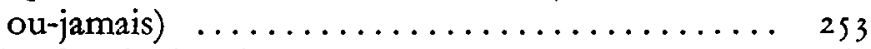

Le Quid et le Quod ................... 265 
